\title{
CONSTRUCTION OF ALGEBRAIC AND DIFFERENCE EQUATIONS WITH A PRESCRIBED SOLUTION SPACE
}

\author{
LAZAROS MOYSIS $^{a, *}$, NICHOLAS P. KARAMPETAKIS ${ }^{a}$ \\ ${ }^{a}$ Department of Mathematics, Faculty of Sciences \\ Aristotle University of Thessaloniki, 54124, Thessaloniki, Greece \\ e-mail: \{lazarosm, karampet\} @math.auth.gr
}

\begin{abstract}
This paper studies the solution space of systems of algebraic and difference equations, given as auto-regressive (AR) representations $A(\sigma) \beta(k)=0$, where $\sigma$ denotes the shift forward operator and $A(\sigma)$ is a regular polynomial matrix. The solution space of such systems consists of forward and backward propagating solutions, over a finite time horizon. This solution space can be constructed from knowledge of the finite and infinite elementary divisor structure of $A(\sigma)$. This work deals with the inverse problem of constructing a family of polynomial matrices $A(\sigma)$ such that the system $A(\sigma) \beta(k)=0$ satisfies some given forward and backward behavior. Initially, the connection between the backward behavior of an AR representation and the forward behavior of its dual system is showcased. This result is used to construct a system satisfying a certain backward behavior. By combining this result with the method provided by Gohberg et al. (2009) for constructing a system with a forward behavior, an algorithm is proposed for computing a system satisfying the prescribed forward and backward behavior.
\end{abstract}

Keywords: algebraic and difference equations, behavior, exact modeling, auto-regressive representation, discrete time system, higher order system.

\section{Introduction}

Let $\mathbb{R}$ be the field of reals, $\mathbb{R}[s]$ the ring of polynomials with coefficients from $\mathbb{R}$ and $\mathbb{R}(s)$ the field of rational functions. By $\mathbb{R}[s]^{p \times m}, \mathbb{R}(s)^{p \times m}, \mathbb{R}_{p r}(s)^{p \times m}$ we denote the sets of $p \times m$ polynomial, rational and proper rational matrices with real coefficients, respectively. We consider systems of higher order discrete time algebraic and difference equations that are described by the matrix equation

$$
A_{q} \beta(k+q)+A_{q-1} \beta(k+q-1)+\cdots+A_{0} \beta(k)=0
$$

or, equivalently,

$$
A(\sigma) \beta(k)=0,
$$

where $k=0,1, \ldots, N-q, A_{0}, \ldots, A_{q} \in \mathbb{R}^{r \times r}, \beta(k)$ : $[0, N] \rightarrow \mathbb{R}^{r}$ is the state of the system, $\sigma$ denotes the forward shift operator, i.e., $\sigma^{\beta(k)}=\beta(k+1)$, and

$$
A(\sigma)=A_{q} \sigma^{q}+\cdots+A_{1} \sigma+A_{0} \in \mathbb{R}[\sigma]^{r \times r}
$$

is a regular polynomial matrix with $\operatorname{det}[A(\sigma)] \neq 0$ and $A_{q} \neq 0, A_{0} \neq 0$. The number $q$ is often called the lag of

\footnotetext{
* Corresponding author
}

the system (Markovsky et al., 2006). Systems described by (2) are called auto-regressive (AR) representations.

Such higher-order systems often appear in systems theory, since they can accurately model many natural or artificial systems, like economic or biological discrete time phenomena. Such examples include the population growth model in biology and the Leontief multisector economy model in economics (Campbell, 1980), as well as other applications in engineering, social sciences and medicine for positive systems (Kaczorek, 2014) or 2D systems (Kaczorek, 2015).

The solution space of (2) consists of both forward and backward solutions and is denoted as

$$
B:=\{\beta(k) \mid(2) \text { is satisfied } \forall k \in[0, N-q]\} .
$$

Forward solutions are defined in the sense that the initial conditions are given and $\beta(k)$ is to be determined in a forward fashion from its previous values. Backward solutions are defined in the sense that the final conditions are given and $\beta(k)$ is to be determined in a backward fashion from its future values.

The solution of such systems has been previously 
studied by various authors (Antoniou et al., 1998; Antsaklis and Michel, 2006; Gohberg et al., 2009; Karampetakis, 2004). A method for constructing the forward (resp. backward) solution space of (2), based on the finite (resp. infinite) elementary divisor structure of $A(\sigma)$, was presented by Gohberg et al. (2009) (resp. Antoniou et al., 1998), whereas the results were extended for non-regular systems by Karampetakis (2004). The algebraic structure of polynomial matrices was studied by Bernstein (2009), Gantmacher (1959), Gohberg et al. (2009), Hayton et al. (1988), Kaczorek (2007), Karampetakis and Vologiannidis (2003), Karampetakis et al. (2004), Praagman (1991), Zaballa and Tisseur (2012), and in the references cited therein.

An interesting problem that we face in this paper is the following inverse problem: Given a certain forward/backward solution space, find a system of algebraic and difference equations with the prescribed solution space. This problem was initially presented and solved by Gohberg et al. (2009), but only for the case of the forward solution space. More specifically, Gohberg et al. (2009) proposed a formula that connects the form of the unknown polynomial matrix $A(\sigma)$ and a finite Jordan pair that can be constructed from the prescribed forward solution space. The main aim of this work is to extend the results presented by Gohberg et al. (2009) for the case where, except from a given forward behavior, a backward behavior is also provided. In order to achieve this, we connect the backward solution space of (2) with the forward solution space of its dual system

$$
\begin{array}{r}
A_{0} \beta(k+q)+A_{1} \beta(k+q-1) \\
+\cdots+A_{q} \beta(k)=0,
\end{array}
$$

and combine this with the results given by Gohberg et al. (2009). This methodology was used for the first time by Karampetakis (2015) for the case of continuous time systems, where the finite and infinite zero structure of $A(\sigma)$ is connected with the smooth and impulsive behavior of (2), respectively.

This paper is organized as follows. In Section 2, some background is provided on the algebraic structure of polynomial matrices. In Section 3, the finite and infinite zero structure of a polynomial matrix is connected to the forward and backward solution space of its corresponding system. Section 4 deals with the inverse problem of modeling the forward and backward solution space of a system, and Section 5 combines the results of the previous sections into a single algorithm. Lastly, in Section 6, following the behavioral framework of Antoulas and Willems (1993), Markovsky et al. (2006), Willems (1986; 1991; 2007), or Zerz (2008a; 2008b; 2011), we study the conditions under which the constructed system is most powerful. Section 7 concludes the paper.

\section{Structural properties of regular polynomial matrices}

In this section we provide some background on the finite and infinite zero structure of polynomial matrices.

Definition 1. (Gantmacher, 1959; Vardulakis, 1991) A square polynomial matrix $A(\sigma) \in \mathbb{R}[\sigma]^{r \times r}$ is called unimodular if $\operatorname{det} A(\sigma)=c \in \mathbb{R}, c \neq 0$. A rational matrix $A(\sigma) \in \mathbb{R}_{p r}(\sigma)^{r \times r}$ is called biproper if $\lim _{\sigma \rightarrow \infty} A(\sigma)=$ $E \in \mathbb{R}^{r \times r}$ with $\operatorname{rank} E=r$

Theorem 1. (Gantmacher, 1959; Vardulakis, 1991) Let $A(\sigma)$ be as in (3). There exist unimodular matrices $U_{L}(\sigma), U_{R}(\sigma) \in \mathbb{R}[\sigma]^{r \times r}$ such that

$$
\begin{aligned}
& S_{A(\sigma)}^{\mathbb{C}}(\sigma) \\
& \quad=U_{L}(\sigma) A(\sigma) U_{R}(\sigma) \\
& \quad=\operatorname{diag}\left(1, \ldots, 1, f_{z}(\sigma), f_{z+1}(\sigma), \ldots, f_{r}(\sigma)\right),
\end{aligned}
$$

with $1 \leq z \leq r$ and $f_{j}(\sigma) \mid f_{j+1}(\sigma)$, for $j=z, z+$ $1, \ldots, r . \quad S_{A(\sigma)}^{\mathbb{C}}(\sigma)$ is called the Smith form of $A(\sigma)$, where $f_{j}(\sigma) \in \mathbb{R}[\sigma]$ are the invariant polynomials of $A(\sigma)$.

The zeros $\lambda_{i} \in \mathbb{C}$ of $f_{j}(\sigma), j=z, z+1, \ldots, r$, are called finite zeros of $A(\sigma)$. Assume that $A(\sigma)$ has $\ell$ finite, distinct zeros. The partial multiplicities $n_{i, j}$ of each zero $\lambda_{i} \in \mathbb{C}, i=1, \ldots, \ell$ satisfy $0 \leq n_{i, z} \leq n_{i, z+1} \leq \cdots \leq$ $n_{i, r}$ with $f_{j}(\sigma)=\left(\sigma-\lambda_{i}\right)^{n_{i, j}} \hat{f}_{j}(\sigma), j=z, \ldots, r$ and $\hat{f}_{j}\left(\lambda_{i}\right) \neq 0$. The terms $\left(\sigma-\lambda_{i}\right)^{n_{i, j}}$ are called finite elementary divisors of $A(\sigma)$ at $\lambda_{i}$. The multiplicity of each zero is defined as $n_{i}=\sum_{j=z}^{r} n_{i, j}$. We denote by $n$ the sum of the degrees of the finite elementary divisors of $A(\sigma)$,

$$
\begin{aligned}
n & :=\operatorname{deg} \operatorname{det} A(\sigma) \\
& =\operatorname{deg}\left(\prod_{j=z}^{r} f_{j}(\sigma)\right)=\sum_{i=1}^{\ell} \sum_{j=z}^{r} n_{i, j} .
\end{aligned}
$$

Similarly, we can find $U_{L}(\sigma) \in \mathbb{R}(\sigma)^{r \times r}, U_{R}(\sigma) \in$ $\mathbb{R}(\sigma)^{r \times r}$ having no poles and zeros at $\sigma=\lambda_{0}$ such that

$$
\begin{aligned}
& S_{A(\sigma)}^{\lambda_{0}}(\sigma) \\
& \quad=U_{L}(\sigma) A(\sigma) U_{R}(\sigma) \\
& \quad=\operatorname{diag}\left(1, \ldots, 1,\left(\sigma-\lambda_{0}\right)^{n_{z}}, \ldots,\left(\sigma-\lambda_{0}\right)^{n_{r}}\right) .
\end{aligned}
$$

$S_{A(\sigma)}^{\lambda_{0}}(\sigma)$ is called the Smith form of $A(\sigma)$ at the local point $\lambda_{0}$.

Theorem 2. (Vardulakis et al., 1982) Let $A(\sigma)$ be as in (3). There exist biproper matrices $U_{L}(\sigma), U_{R}(\sigma) \in$ $\mathbb{R}_{p r}(\sigma)^{r \times r}$ such that

$$
S_{A(\sigma)}^{\infty}(\sigma)
$$




$$
\begin{aligned}
& =U_{L}(\sigma) A(\sigma) U_{R}(\sigma) \\
& =\operatorname{diag}(\underbrace{\sigma^{q_{1}}, \ldots, \sigma^{q_{u}}}_{u}, \overbrace{\frac{1}{\sigma^{\hat{q}_{u+1}}}, \frac{1}{\sigma^{\hat{q}_{u+2}}}, \ldots, \frac{1}{\sigma^{\hat{q}_{r}}}}^{r-u}),
\end{aligned}
$$

with

$$
q_{1} \geq \ldots \geq q_{u} \geq 0, \quad \hat{q}_{r} \geq \hat{q}_{r-1} \geq \ldots \hat{q}_{u+1}>0,
$$

and $1 \leq u \leq r . S_{\mathrm{A}(\sigma)}^{\infty}(\sigma)$ is called the Smith form of $A(\sigma)$ at infinity.

If $p_{\infty}$ is the number of $q_{i}$ 's in (9) with $q_{i}>0$, then we say that $A(\sigma)$ has $p_{\infty}$ poles at infinity, each one of order $q_{i}>0$. Also, if $z_{\infty}$ is the number of $\hat{q}_{i}$ 's in (9), then we say that $A(\sigma)$ has $z_{\infty}$ zeros at infinity, each one of order $\hat{q}_{i}>0$.

Lemma 1. (Vardulakis, 1991, Corollary 3.54) For $A(\sigma)$ in (3) we have $q_{1}=q$

Definition 2. (Vardulakis, 1991, Section 4.2.1) The dual polynomial matrix of $A(\sigma)$ is defined as

$$
\tilde{\mathrm{A}}(\sigma):=\sigma^{q} A\left(\frac{1}{\sigma}\right)=A_{0} \sigma^{q}+A_{1} \sigma^{q-1}+\cdots+A_{q} .
$$

Theorem 3. (Vardulakis, 1991, Section 4.2.1) Let $\tilde{A}(\sigma)$ be as in (10). There exist matrices $\tilde{U}_{L}(\sigma) \in \mathbb{R}(\sigma)^{r \times r}$, $\tilde{U}_{R}(\sigma) \in \mathbb{R}(\sigma)^{r \times r}$ having no poles or zeros at $\sigma=0$, such that

$$
\begin{aligned}
S_{\tilde{A}(\sigma)}^{0}(\sigma) & =\tilde{U}_{L}(\sigma) \tilde{A}(\sigma) \tilde{U}_{R}(\sigma) \\
& =\operatorname{diag}\left(\sigma^{\mu_{1}}, \ldots, \sigma^{\mu_{r}}\right),
\end{aligned}
$$

$S_{\tilde{A}(\sigma)}^{0}(\sigma)$ being the local Smith form of $\tilde{A}(\sigma)$ at $\sigma=0$.

The terms $\sigma^{\mu_{j}}$ are the finite elementary divisors of $\tilde{A}(\sigma)$ at zero and are called the infinite elementary divisors (i.e.d.) of $A(\sigma)$.

The connection between the Smith form at the infinity of $A(\sigma)$ and the Smith form at the zero of the dual matrix is given by Hayton et al. (1988) and Vardulakis (1991):

$$
\begin{aligned}
& S_{\tilde{A}(\sigma)}^{0}(\sigma) \\
& =\operatorname{diag}\left(\sigma^{\mu_{1}}, \ldots, \sigma^{\mu_{r}}\right) \\
& =\operatorname{diag}(1, \underbrace{\sigma^{q-q_{2}}, \ldots, \sigma^{q-q_{u}}}_{\text {i.p.e.d. }}, \underbrace{\sigma^{q+\hat{q}_{u+1}}, \ldots, \sigma^{q+\hat{q}_{r}}}_{\text {i.z.e.d. }}),
\end{aligned}
$$

where by i.p.e.d. and i.z.e.d. we denote the infinite pole and infinite zero elementary divisors, respectively. From the above formula it can be seen that the the orders of the infinite elementary divisors of $A(\sigma)$ are given by

$$
\mu_{1}=q-q_{1} \stackrel{q=q_{1}}{=} 0,
$$

$$
\begin{array}{ll}
\mu_{j}=q-q_{j}, & j=2,3, \ldots, u, \\
\mu_{j}=q+\hat{q}_{j}, & j=u+1, \ldots, r .
\end{array}
$$

We denote by $\mu$ the sum of the degrees of the infinite elementary divisors of $A(\sigma)$, i.e.,

$$
\mu:=\sum_{j=1}^{r} \mu_{j} .
$$

Lemma 2. (Antoniou et al., 1998; Gohberg et al., 2009) Let $A(\sigma)$ be as in (3). Let also $n$ and $\mu$ be the sums of the degrees of the finite and infinite elementary divisors of $A(\sigma)$, respectively, as defined in (7) and (14). Then

$$
n+\mu=r \times q .
$$

The above relation is of fundamental importance, since it will help us determine whether or not the construction of a system with a prescribed behavior is possible.

It should be noted that in the case where the matrix $A(\sigma)$ is non-regular, that is, $A(\sigma) \in \mathbb{R}[\sigma]^{r \times m}$ and $r \neq m$ or $A(\sigma) \in \mathbb{R}[\sigma]^{r \times r}$ and $\operatorname{det} A(\sigma)=0$, the algebraic structure of $A(\sigma)$ and by extension the solution space of (2) are connected with additional invariants due to the left and right null space of $A(\sigma)$ (see Karampetakis, 2004; Praagman, 1991).

\section{Jordan pairs and solutions of $A(\sigma) \beta(k)=0$}

In this section we will connect the finite and infinite zero structure of the polynomial matrix $A(\sigma)$ with the forward and backward behavior of the system (2).

\subsection{Finite Jordan pairs and the forward solution} space.

Definition 3. (Antoniou et al., 1998; Gohberg et al., 2009) Let $\left(C_{i} \in \mathbb{C}^{r \times n_{i}}, J_{i} \in \mathbb{C}^{n_{i} \times n_{i}}\right)$ be a matrix pair, where $J_{i}$ is in Jordan form, corresponding to the zero of $\lambda_{i} \in$ $\mathbb{C}$ of $A(\sigma)$ with multiplicity $n_{i}$. That is, $J_{i}$ consists of Jordan blocks with sizes equal to the partial multiplicities of $\lambda_{i}$. This is called a finite Jordan pair of $A(\sigma)$ (or a finite eigenpair) corresponding to $\lambda_{i}$ iff

1. $\operatorname{det} A(\sigma)$ has a zero at $\lambda_{i}$ of multiplicity $n_{i}$,

2. $\operatorname{rankcol}\left(C_{i} J_{i}^{k}\right)_{k=0}^{q-1}=n_{i}$,

3. $\sum_{k=0}^{q} A_{k} C_{i} J_{i}^{k}=0$.

Taking an eigenpair for each distinct finite zero $\lambda_{i}$, $i=1, \ldots, \ell$, of $A(\sigma)$, we can create the finite spectral pair of $A(\sigma)$, i.e., $C_{F} \in \mathbb{C}^{r \times n}, J_{F} \in \mathbb{C}^{n \times n}$, where

$$
\begin{aligned}
C_{F} & =\left(C_{1}, \ldots, C_{\ell}\right), \\
J_{F} & =\operatorname{blockdiag}\left(J_{1}, \ldots, J_{\ell}\right) .
\end{aligned}
$$


The finite spectral pair satisfies similar properties, i.e.,

$$
\begin{array}{r}
\operatorname{deg}(\operatorname{det} A(\sigma))=n, \\
\operatorname{rankcol}\left(C_{F} J_{F}{ }^{k}\right)_{k=0}^{q-1}=n, \\
\sum_{k=0}^{q} A_{k} C_{F} J_{F}^{k}=0 .
\end{array}
$$

Theorem 4. (Antoniou et al., 1998; Gohberg et al., 2009) The forward solution space $B_{F}$ of (2) is given by the column span of the matrix

$$
\Psi_{F}=\left\langle C_{F} J_{F}^{k}\right\rangle
$$

and has dimension $\operatorname{dim} B_{F}=n$.

\subsection{Infinite Jordan pairs and the backward solution space.}

Definition 4. (Antoniou et al., 1998; Gohberg et al., 2009) An eigenpair of the dual matrix $\tilde{A}(\sigma)$ corresponding to the eigenvalue $\tilde{\lambda}=0$ is called an infinite eigenpair of $A(\sigma)$ (or an infinite Jordan pair). Taking an eigenpair for each finite zero $\tilde{\lambda}=0$ of $\tilde{A}(\sigma)$, we construct the infinite spectral pair of $A(\sigma)$,

$$
\begin{gathered}
C_{\infty}=\left(C_{\infty, \mu_{1}}, \ldots, C_{\infty, \mu_{r}}\right) \\
J_{\infty}=\operatorname{blockdiag}\left(J_{\infty, \mu_{1}}, \ldots, J_{\infty, \mu_{r}}\right),
\end{gathered}
$$

which satisfies the following:

1. $\operatorname{det} \tilde{A}(\sigma)$ has a zero at $\tilde{\lambda}=0$ of multiplicity $\mu$,

2. $\operatorname{rankcol}\left(C_{\infty} J_{\infty}^{k}\right)_{k=0}^{q-1}=\mu$,

3. $\sum_{k=0}^{q} A_{k} C_{\infty} J_{\infty}^{q-k}=0$.

Theorem 5. (Antoniou et al., 1998) The backward solution space $B_{B}$ of (2) is given by the column span of the matrix

$$
\Psi_{B}=\left\langle C_{\infty} J_{\infty}^{N-k}\right\rangle,
$$

and has dimension $\operatorname{dim} B_{B}=\mu$.

In the following we shall provide an analytic formula for the backward solution space.

\section{Construction of a system with a given backward behavior}

In the previous section we have provided a method for constructing the complete forward and backward solution space of (2), when knowledge of the finite and infinite Jordan pairs of the matrix $A(\sigma)$ is available. If the Jordan pairs of $A(\sigma)$ are not given beforehand, a method for constructing them can be found in the works of
Gohberg et al. (2009) and Karampetakis (2004). In the following sections we study the inverse problem, that is: Given a specific forward or backward behavior, how to construct a polynomial matrix $A(\sigma)$ and its corresponding homogenous system $A(\sigma) \beta(k)=0$ that will satisfy the given behavior.

An answer to this modeling problem was first proposed by Gohberg et al. (2009), for the case of the forward solution space. We first present these results and then extend them in order to include the case of the backward solution space as well. In the next section, we will combine these results into a single algorithm for modelling both the forward and the backward solution space of a system.

Suppose that a finite number of vector valued functions $\beta_{i}(k):[0, N] \rightarrow \mathbb{R}^{r}$ of the form

$$
\begin{aligned}
\beta_{i}(k):= & \lambda_{i}^{k} \beta_{i, n_{i}-1}+k \lambda_{i}^{k-1} \beta_{i, n_{i}-2} \\
& +\cdots+\left(\begin{array}{c}
k \\
n_{i}-1
\end{array}\right) \lambda_{i}^{k-\left(n_{i}-1\right)} \beta_{i, 0},
\end{aligned}
$$

for $\lambda_{i} \neq 0, i=1,2, \ldots, \ell$, or

$$
\beta_{i}(k)=\delta(k) \beta_{i, n_{i}-1}+\cdots+\delta\left(k-\left(n_{i}-1\right)\right) \beta_{i, 0},
$$

for $\lambda_{i}=0$, are given, where $\delta(k)$ denotes the discrete Kronecker delta function and $\beta_{i, 0}, \ldots, \beta_{i, n_{i}-1} \in \mathbb{C}^{r}$. We want to construct a system of difference equations with (23), (24) as its solutions. By analogy to the continuous time case studied by Gohberg et al. (2009, Proposition 1.9), if 23, (24) are solutions of the system, then the vectors $\beta_{i, 0}, \ldots, \beta_{i, n_{i}-1}$ are generalized eigenvectors of $A(\sigma)$ corresponding to $\lambda_{i}$ and the matrices $\left(C_{i}, J_{i}\right)$, with

$$
\begin{aligned}
C_{i} & =\left(\begin{array}{cccc}
\beta_{i, 0} & \ldots & \beta_{i, n_{i}-1}
\end{array}\right) \\
J_{i} & =\left(\begin{array}{cccc}
\lambda_{i} & 1 & \ldots & 0 \\
0 & \lambda_{i} & \ddots & \vdots \\
\vdots & \ddots & \ddots & 1 \\
0 & \ldots & 0 & \lambda_{i}
\end{array}\right),
\end{aligned}
$$

constitute a Jordan pair of $A(\sigma)$. Thus, the vector functions

$$
\beta_{i, 0} \lambda_{i}^{k}, \quad \beta_{i, 0} k \lambda_{i}^{k-1}+\beta_{i, 1} \lambda_{i}^{k}, \ldots
$$

for $\lambda_{i} \neq 0$ and

$$
\beta_{i, 0} \delta(k), \quad \beta_{i, 0} \delta(k-1)+\beta_{i, 1} \delta(k), \ldots
$$

respectively for $\lambda_{i}=0$ are also solutions of the system (Gohberg et al., 2009, Section 8.3). The above set of vector valued functions can be written in matrix form as 
$C_{i} J_{i}^{k}$, where we make use of the formulas

$J_{i}^{k}=\left(\begin{array}{cccc}\lambda_{i}^{k} & \left(\begin{array}{c}k \\ 1\end{array}\right) \lambda_{i}^{k-1} & \cdots & \left(\begin{array}{c}k \\ n_{i}-1\end{array}\right) \lambda_{i}^{k-\left(n_{i}-1\right)} \\ 0 & \lambda_{i}^{k} & \cdots & \left(\begin{array}{c}k \\ n_{i}-2\end{array}\right) \lambda_{i}^{k-\left(n_{i}-2\right)} \\ \vdots & & \ddots & \vdots \\ 0 & \cdots & \cdots & \lambda_{i}^{k}\end{array}\right), \quad \lambda_{i} \neq 0$,

$J_{i}^{k}=\left(\begin{array}{cccc}\delta(k) & \delta(k-1) & \cdots & \delta\left(k-\left(n_{i}-1\right)\right) \\ 0 & \delta(k) & \cdots & \delta\left(k-\left(n_{i}-2\right)\right) \\ \vdots & & \ddots & \vdots \\ 0 & \cdots & \cdots & \delta(k)\end{array}\right), \quad \lambda_{i}=0$,

with $C_{i} \in \mathbb{C}^{r \times n_{i}}, J_{i} \in \mathbb{C}^{n_{i} \times n_{i}}$. Define

$$
\begin{gathered}
C:=\left(\begin{array}{ccc}
C_{1} & \cdots & C_{\ell}
\end{array}\right) \in \mathbb{C}^{r \times n}, \\
J:=\operatorname{blockdiag}\left(J_{1}, \ldots, J_{\ell}\right) \in \mathbb{C}^{n \times n},
\end{gathered}
$$

where $n:=\sum_{i=1}^{\ell} n_{i}$. Then we have the following theorem.

Theorem 6. (Gohberg et al., 2009) Let a be a complex number different from $\lambda_{i}$ and define

$$
\begin{aligned}
A(\sigma)= & I_{r}-C\left(J-a I_{n}\right)^{-q}\left((\sigma-a) V_{q}\right. \\
& \left.+\cdots+(\sigma-a)^{q} V_{1}\right),
\end{aligned}
$$

where $q=\operatorname{ind}(C, J)$ is the least integer such that the matrix

$$
S_{q-1}=\left(\begin{array}{c}
C \\
C J \\
\vdots \\
C J^{q-1}
\end{array}\right) \in \mathbb{C}^{r q \times n}
$$

has full column rank, and $\left(V_{1}, \ldots, V_{q}\right)$ is the generalized inverse of

$$
S_{1-q}=\left(\begin{array}{c}
C \\
C\left(J-a I_{n}\right)^{-1} \\
\vdots \\
C\left(J-a I_{n}\right)^{1-q}
\end{array}\right) \in \mathbb{C}^{r q \times n} .
$$

Then $\beta_{i}(k)$ are solutions of (2). Furthermore, $q$ is the minimal possible lag of any polynomial matrix with this property.

Remark 1. Different choices of $a \neq \lambda_{i}$ will lead to the construction of matrices that are left unimodular equivalent. That is, if $A_{1}(\sigma)$ and $A_{2}(\sigma)$ are any two matrices that are constructed for different values of the parameter $a$, there exists a unimodular matrix $U(\sigma)$ such that

$$
A_{1}(\sigma)=U(\sigma) A_{2}(\sigma) .
$$

Thus, since multiplication by $U(\sigma)$ does not alter the algebraic structure of $A(\sigma)$, the behavior of the corresponding system remains the same. In consequence, the choice of the parameter $a$ does not impose any limitations.
Notice that since the time sequences in the form of (23) constitute solutions to (2), they satisfy (2) for all $k$, regardless of how we consider time propagation. This means that solutions in the form of 23) can either be regarded as solutions propagating forward in time for initial conditions $\beta(0), \beta(1), \ldots, \beta(q-1)$, or solutions moving backward for the final conditions $\beta(N), \beta(N-$ $1), \ldots, \beta(N-q+1)$.

Remark 2. In the case where a backward propagating time sequence is given in the form of $\beta_{i}(k)=$ $C_{i} J_{i}^{N-k} x_{N}$, corresponding to a zero $\lambda_{i} \neq 0$, with the final conditions

$$
\left(\begin{array}{c}
\beta_{i}(N) \\
\vdots \\
\beta_{i}(N-q+1)
\end{array}\right)=\left(\begin{array}{c}
C_{i} \\
\vdots \\
C_{i} J_{i}^{q-1}
\end{array}\right) x_{N}
$$

it can be rewritten as

$$
\beta_{i}(k)=C_{i}\left(J_{i}^{-1}\right)^{k} \underbrace{J_{i}^{N} x_{N}}_{x_{0}}
$$

and it can be clearly seen that it corresponds to the finite Jordan pair $\left(C, J^{-1}\right)$ of $A(\sigma)$ for the initial conditions

$$
\left(\begin{array}{c}
\beta_{i}(0) \\
\vdots \\
\beta_{i}(q-1)
\end{array}\right)=\left(\begin{array}{c}
C_{i} \\
\vdots \\
C_{i}\left(J_{i}^{-1}\right)^{q-1}
\end{array}\right) x_{0}
$$

Therefore, in order to construct the system (2) with $\beta_{i}(k)$ as its solution, one can follow Theorem 6 using the pair $\left(C, J^{-1}\right)$.

On the other hand, time sequences in the form of 24 can only be considered forward solutions for the system.

Overall, the finite elementary divisors are connected to either forward/backward propagating solutions of the form (23) for a non-zero $\lambda_{i} \neq 0$, or to strictly forward propagating solutions of the form (24) for $\lambda_{i}=0$.

In the following, we will show how the infinite elementary divisors are connected to the backward solutions of the system.

What we need to note here is that although we have created an auto-regressive representation for the given forward solution space, if the equation $n+\mu=r \times q$ is not satisfied for $\mu=0$, then the above algorithm will give rise to an AR the representation which will include some extra forward/backward behavior. An example showcasing this is given in the last section.

Now we will provide a theorem for the backward solution space.

Theorem 7. (Karampetakis, 2004) Let $\tilde{A}(\sigma)$ be the dual matrix of $A(\sigma)$, as defined in (10). From (11), let

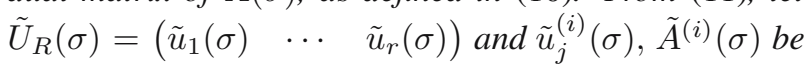


the $i$-th derivatives of $\tilde{u}_{j}(\sigma)$ and $\tilde{A}(\sigma)$ for $j=2, \ldots, r$ and $i=0,1, \ldots, \mu_{j}-1$, with $\mu_{j}$ defined in 13. Define

$$
x_{j, i}=\frac{1}{i !} \tilde{u}_{j}^{(i)}(\sigma) .
$$

Then the discrete time vector valued functions

$$
\begin{aligned}
\beta_{j}(k)= & x_{j, \mu_{j}-1} \delta(N-k)+\ldots \\
& +x_{j, 0} \delta\left(N-k-\mu_{j}+1\right)
\end{aligned}
$$

are linearly independent backward solutions of $A(\sigma) \beta(k)=0$.

Lemma 3. (Karampetakis, 2015; Vardulakis, 1991) The vectors $x_{j, i}$, defined in (36) for $i=0,1, \ldots, \mu_{j}-1$ and $j=2, \ldots, r$, form Jordan chains for $\tilde{A}(\sigma)$ corresponding to the eigenvalue $\tilde{\lambda}_{i}=0$ with lengths $\mu_{j}$, as in 13. Thus, they satisfy the following systems of equations:

$$
\left(\begin{array}{ccc}
A_{q} & \cdots & 0 \\
\vdots & \ddots & \vdots \\
A_{q_{j}+1} & \cdots & A_{q}
\end{array}\right)\left(\begin{array}{c}
x_{j, 0} \\
\vdots \\
x_{j, q-q_{j}-1}
\end{array}\right)=\left(\begin{array}{c}
0 \\
\vdots \\
0
\end{array}\right)
$$

for the case of infinite pole elementary divisors, i.e., $\mu_{j}=$ $q-q_{j}, \quad j=2, \ldots, u$, and

$$
\underbrace{\left(\begin{array}{cccccc}
A_{q} & 0 & 0 & \cdots & \cdots & 0 \\
\vdots & A_{q} & 0 & \cdots & \cdots & 0 \\
A_{0} & \vdots & \ddots & \ddots & \ddots & \vdots \\
0 & A_{0} & \ddots & \ddots & \ddots & \vdots \\
\vdots & \ddots & \ddots & \ddots & \ddots & 0 \\
0 & \cdots & 0 & A_{0} & \cdots & A_{q}
\end{array}\right)}_{Q}\left(\begin{array}{c}
x_{j, 0} \\
\vdots \\
x_{j, q+\hat{q}_{j}-1}
\end{array}\right)=\left(\begin{array}{c}
0 \\
\vdots \\
0
\end{array}\right)
$$

for the case of infinite zero elementary divisors, i.e., $\mu_{j}=$ $q+\hat{q}_{j}, \quad j=u+1, \ldots, r$.

The proof of (39) that corresponds to the infinite zero elementary divisors was provided by Vardulakis (1991). The procedure can be easily extended with no significant changes to include the case of infinite pole elementary divisors 38 .

The following theorem showcases the connection between the backward solutions of (2) and the forward solutions of the dual system (5)

Theorem 8. Let $x_{j, i}$ be the vectors defined in (36) for $i=0,1, \ldots, \mu_{j}-1$ and $j=2, \ldots, r$. The vector valued function (37) is a solution of the AR representation (2) iff the vector

$$
\tilde{\beta}_{j}(k)=x_{j, 0} \delta\left(k-\mu_{j}+1\right)+\cdots+x_{j, \mu_{j}-1} \delta(k)
$$

is a solution of the dual system (5), i.e., $\tilde{A}(\sigma) \tilde{\beta}_{j}(k)=0$.
Proof. With no loss of generality, we shall study the case of $\mu_{j}=q+\hat{q}_{j}$. The proof of $\mu_{j}=q-q_{j}$ proceeds in a similar manner.

(Necessity) First we will prove that if $\beta_{j}(k)$ is a solution of (2) then $\tilde{\beta}_{j}(k)$ is a solution of (5).

Let $\tilde{B}(z)=\mathcal{Z}[\tilde{\beta}(k)]=\sum_{k=0}^{N} z^{-k} \beta(k)$ be the $\mathcal{Z}$-transform of $\tilde{\beta}(k)$. Taking $\mathcal{Z}$-transforms on the dual system, we have

$$
\mathcal{Z}[\tilde{A}(\sigma) \tilde{\beta}(k)]=0 \Rightarrow \tilde{A}(z) \tilde{B}(z)=\tilde{\beta}_{i n},
$$

where

$$
\begin{aligned}
& \tilde{\beta}_{i n}:=\left(\begin{array}{lll}
z^{q} I_{r} & \cdots & z I_{r}
\end{array}\right) \\
& \times\left(\begin{array}{ccc}
A_{0} & \cdots & 0 \\
\vdots & \ddots & \vdots \\
A_{q-1} & \cdots & A_{0}
\end{array}\right)\left(\begin{array}{c}
\tilde{\beta}(0) \\
\vdots \\
\tilde{\beta}(q-1)
\end{array}\right) .
\end{aligned}
$$

The $\mathcal{Z}$ transform of $\tilde{\beta}_{j}(k)$ in 40 is

$$
\tilde{B}_{j}(z)=x_{j, 0} z^{-q-\hat{q}_{j}+1}+\cdots+x_{j, q+\hat{q}_{j}-1} .
$$

Substituting $\tilde{\beta}(z)$ in (41) we obtain

$$
\begin{aligned}
& \tilde{A}(z) \tilde{B}_{j}(z) \\
& =\left(z^{q} A_{0}+\cdots+A_{q}\right)\left(x_{j, 0} z^{-q-\hat{q}_{j}+1}+\cdots+x_{j, q+\hat{q}_{j}-1}\right) \\
& =\left(\begin{array}{llllll}
z^{q} I_{r} & \cdots & I_{r} & z^{-1} I_{r} & \cdots & z^{-q-\hat{q}_{j}+1} I_{r}
\end{array}\right) \\
& \times\left(\begin{array}{ccc}
A_{0} & \cdots & 0 \\
\vdots & \ddots & \vdots \\
A_{q} & \ddots & A_{0} \\
\vdots & \ddots & \vdots \\
0 & \cdots & A_{q}
\end{array}\right)\left(\begin{array}{c}
x_{j, q+\hat{q}_{j}-1} \\
\vdots \\
x_{j, 0}
\end{array}\right) .
\end{aligned}
$$

Thus

$$
\begin{aligned}
& \tilde{A}(z) \tilde{B}_{j}(z) \\
& =\left(\begin{array}{lll}
z^{q} I_{r} & \cdots & z I_{r}
\end{array}\right)\left(\begin{array}{ccc}
A_{0} & \cdots & 0 \\
\vdots & \ddots & \vdots \\
A_{q-1} & \cdots & A_{0}
\end{array}\right)\left(\begin{array}{c}
x_{j, q+\hat{q}_{j}-1} \\
\vdots \\
x_{j, \hat{q}_{j}}
\end{array}\right) \\
& +\left(\begin{array}{lll}
I_{r} & \cdots & z^{-q-\hat{q}_{j}+1} I_{r}
\end{array}\right) \\
& \underbrace{Q^{T}}_{=0 \text { (see Lemma } 3}
\end{aligned}
$$

Hence

$$
\begin{aligned}
& \tilde{A}(z) \tilde{B}_{j}(z)=\left(\begin{array}{lll}
z^{q} I_{r} & \cdots & z I_{r}
\end{array}\right) \\
& \times\left(\begin{array}{ccc}
A_{0} & \cdots & 0 \\
\vdots & \ddots & \vdots \\
A_{q-1} & \cdots & A_{0}
\end{array}\right)\left(\begin{array}{c}
x_{j, q+\hat{q}_{j}-1} \\
\vdots \\
x_{j, \hat{q}_{j}}
\end{array}\right)
\end{aligned}
$$


We can easily check that (41) and (46) coincide in the case where

$$
\left(\begin{array}{c}
\tilde{\beta}_{j}(0) \\
\vdots \\
\tilde{\beta}_{j}(q-1)
\end{array}\right)=\left(\begin{array}{c}
x_{j, q+\hat{q}_{j}-1} \\
\vdots \\
x_{j, \hat{q}_{j}}
\end{array}\right) .
$$

Therefore, $\tilde{\beta}_{j}(k)$ is a solution of (5) for the above initial conditions.

(Sufficiency) Now we will show the opposite, that is, if $\tilde{\beta}_{j}(k)$ is a solution of (5) then $\beta_{j}(k)$ is a solution of (2).

Since $\tilde{\beta}_{j}(k)$ is a solution of $[5$, we shall have that

$$
\begin{aligned}
& \tilde{A}(z) \tilde{B}_{j}(z) \\
& =\left(z^{q} A_{0}+\cdots+A_{q}\right)\left(x_{j, 0} z^{-q-\hat{q}_{j}+1}+\cdots+x_{j, q+\hat{q}_{j}-1}\right) \\
& =\left(\begin{array}{lll}
z^{q} I_{r} & \cdots & z I_{r}
\end{array}\right)\left(\begin{array}{ccc}
A_{0} & \cdots & 0 \\
\vdots & \ddots & \vdots \\
A_{q-1} & \cdots & A_{0}
\end{array}\right)\left(\begin{array}{c}
\tilde{\beta}_{j}(0) \\
\vdots \\
\tilde{\beta}_{j}(q-1)
\end{array}\right) \cdot(48
\end{aligned}
$$

The above equation holds true if and only if the proper part on the left-hand side of the above equation, cf. (45),

$$
\left(\begin{array}{lllll}
z^{-q-\hat{q}_{j}+1} I_{r} & \cdots & z^{-q} I_{r} & \cdots & I_{r}
\end{array}\right) Q\left(\begin{array}{c}
x_{j, 0} \\
\vdots \\
x_{j, q+\hat{q}_{j}-1}
\end{array}\right)
$$

is equal to zero. This holds true if and only if the condition (39) is satisfied. However, according to Lemma 3 if (39) is satisfied then $\beta_{j}(k)$ is a solution of (2).

The previous theorem proves that the problem of finding an AR representation of the form (2) with the following backward behavior:

$$
\begin{aligned}
\beta_{j}(k)= & x_{j, \mu_{j}-1} \delta(N-k)+\ldots \\
& +x_{j, 0} \delta\left(N-k-\mu_{j}+1\right)
\end{aligned}
$$

is equivalent to that of finding an $\mathrm{AR}$ representation of the form (5) satisfying the forward behavior

$$
\tilde{\beta}_{j}(k)=x_{j, 0} \delta\left(k-\mu_{j}+1\right)+\cdots+x_{j, \mu_{j}-1} \delta(k) .
$$

However, this problem can easily be solved using Theorem 6 This is demonstrated in the following.

Theorem 9. Suppose that the following l vector valued functions:

$$
\beta_{j}(k)=\sum_{w=0}^{\mu_{j}-1} x_{j, \mu_{j}-1-w} \delta(N-w-k)
$$

are given, where $x_{j, 0}, \ldots, x_{j, \mu_{j}-1} \in \mathbb{R}^{r}, j=1, \ldots, l$. Define

$$
C_{j}=\left(\begin{array}{lll}
x_{j, 0} & \cdots & x_{j, \mu_{j}-1}
\end{array}\right) \in \mathbb{R}^{r \times \mu_{j}},
$$

$$
J_{j}=\left(\begin{array}{cccc}
0 & 1 & \cdots & 0 \\
0 & 0 & \ddots & \vdots \\
\vdots & \ddots & \ddots & 1 \\
0 & \cdots & 0 & 0
\end{array}\right) \in \mathbb{R}^{\mu_{j} \times \mu_{j}},
$$

where $j=1,2, \ldots, l$. Let

$$
\begin{gathered}
C=\left(\begin{array}{lll}
C_{1} & \cdots & C_{l}
\end{array}\right) \in \mathbb{R}^{r \times \mu}, \\
J=\operatorname{blockdiag}\left(J_{1}, \ldots, J_{l}\right) \in \mathbb{R}^{\mu \times \mu},
\end{gathered}
$$

with $\mu=\sum_{j=1}^{l} \mu_{j}$. Let $a \neq 0$ and define

$$
\begin{aligned}
\tilde{A}(\sigma)= & I_{r}-C\left(J-a I_{r}\right)^{-q}\left((\sigma-a) V_{q}\right. \\
& \left.+\cdots+(\sigma-a)^{q} V_{1}\right),
\end{aligned}
$$

where $q=\operatorname{ind}(C, J)$ is the least integer such that the matrix

$$
S_{q-1}=\left(\begin{array}{c}
C \\
C J \\
\vdots \\
C J^{q-1}
\end{array}\right)
$$

has full column rank and $V=\left(V_{1}, \ldots, V_{q}\right)$ is the generalized inverse of

$$
S_{1-q}=\left(\begin{array}{c}
C \\
C\left(J-a I_{n}\right)^{-1} \\
\vdots \\
C\left(J-a I_{n}\right)^{1-q}
\end{array}\right) .
$$

Then $\beta_{j}(k)$ are solutions of (21), where $A(\sigma)=\sigma^{q} \tilde{A}\left(\frac{1}{\sigma}\right)$. Furthermore, $q$ is the minimal possible degree of any $r \times r$ polynomial matrix with this property.

Example 1. We want to find a polynomial matrix $A(\sigma)$ such that the system $A(\sigma) \beta(k)=0$ has the backward solution

$$
\begin{aligned}
\beta_{1}(k) & \underbrace{\left(\begin{array}{l}
0 \\
1
\end{array}\right)}_{x_{1,3}} \delta(N-k)+\underbrace{\left(\begin{array}{c}
-1 \\
0
\end{array}\right)}_{x_{1,2}} \delta(N-k-1) \\
& +\underbrace{\left(\begin{array}{l}
1 \\
1
\end{array}\right)}_{x_{1,1}} \delta(N-k-2)+\underbrace{\left(\begin{array}{l}
1 \\
0
\end{array}\right)}_{x_{1,0}} \delta(N-k-3) .
\end{aligned}
$$

We begin by forming the matrices

$$
\begin{aligned}
& C=\left(\begin{array}{llll}
x_{1,0} & x_{1,1} & x_{1,2} & x_{1,3}
\end{array}\right)=\left(\begin{array}{cccc}
1 & 1 & -1 & 0 \\
0 & 1 & 0 & 1
\end{array}\right), \\
& J=\left(\begin{array}{llll}
0 & 1 & 0 & 0 \\
0 & 0 & 1 & 0 \\
0 & 0 & 0 & 1 \\
0 & 0 & 0 & 0
\end{array}\right) .
\end{aligned}
$$


Then, we start assuming values for $q$. Starting from $q=1$, the matrix $S_{1-1}=S_{0}=C$ does not have full column rank. For $q=2$ the matrix

$$
S_{2-1}=S_{1}=\left(\begin{array}{c}
C \\
C J
\end{array}\right)=\left(\begin{array}{cccc}
1 & 1 & -1 & 0 \\
0 & 1 & 0 & 1 \\
\hline 0 & 1 & 1 & -1 \\
0 & 0 & 1 & 0
\end{array}\right)
$$

has full column rank, and thus $A(\sigma)$ has $\operatorname{lag} q=2$. Let $a=1$ and

$$
\begin{aligned}
V & =\left(V_{1} \mid V_{2}\right)=\left(\begin{array}{c}
C \\
C\left(J-I_{4}\right)^{-1}
\end{array}\right)^{-1} \\
& =\left(\begin{array}{cc|cc}
1 & -2 & 0 & -1 \\
-\frac{1}{2} & \frac{3}{2} & -\frac{1}{2} & 1 \\
-\frac{1}{2} & -\frac{1}{2} & -\frac{1}{2} & 0 \\
\frac{1}{2} & -\frac{1}{2} & \frac{1}{2} & -1
\end{array}\right)
\end{aligned}
$$

Thus,

$$
\begin{aligned}
\tilde{A}(\sigma) & =I_{2}-C\left(J-I_{4}\right)^{-2}\left((\sigma-1) V_{2}+(\sigma-1)^{2} V_{1}\right) \\
& =\left(\begin{array}{cc}
\sigma & 2 \sigma^{2}-\sigma-1 \\
-\frac{1}{2} \sigma^{2}+\frac{1}{2} \sigma & \frac{3}{2} \sigma^{2}-\frac{1}{2}
\end{array}\right),
\end{aligned}
$$

with Smith form at zero,

$$
S_{\tilde{A}(\sigma)}^{0}(\sigma)=\left(\begin{array}{cc}
1 & 0 \\
0 & \sigma^{4}
\end{array}\right) .
$$

Therefore, the matrix that we are looking for is

$$
\begin{aligned}
A(\sigma) & =\sigma^{2} \tilde{A}\left(\frac{1}{\sigma}\right) \\
& =\left(\begin{array}{cc}
\sigma & -\sigma^{2}-\sigma+2 \\
\frac{1}{2} \sigma-\frac{1}{2} & -\frac{1}{2} \sigma^{2}+\frac{3}{2}
\end{array}\right),
\end{aligned}
$$

with $\operatorname{det} A(\sigma)=1$ and Smith form at infinity,

$$
S_{\mathrm{A}(\sigma)}^{\infty}(\sigma)=\sigma^{2} S_{\tilde{A}(\sigma)}^{0}\left(\frac{1}{\sigma}\right)=\left(\begin{array}{cc}
\sigma^{2} & 0 \\
0 & \frac{1}{\sigma^{2}}
\end{array}\right) .
$$

\section{Construction of a system with given forward and backward behaviors}

In this section, we combine all the results presented in previous sections, in order to create an algorithm for constructing a system that satisfies both given forward and backward behaviors. We have already presented methods for constructing a system with a given either forward or backward behavior. In the last case, we have constructed a dual polynomial matrix $\tilde{A}(\sigma)$ with a forward behavior resulting from a given backward behavior. Our aim is to work in a similar way for the forward behavior as well, i.e., to connect it to the behavior in the dual system. We can either connect the forward behavior of (2) to a forward or a backward behavior of the dual system (5). We shall work with the first result, so that the problem of finding a system with a given forward-backward behavior is reduced to that of finding its dual system which exhibits a certain forward behavior.

First we outline the connection between the forward behavior of (2) and a corresponding forward behavior of the dual system (5).

Theorem 10. Let $A(\sigma)$ be defined as in (3) with $\operatorname{rank}_{R(\sigma)} A(\sigma)=r$. If $\beta_{j}(k)=C_{j} J_{j}^{k} x_{0}$ is a solution of (2), where $\left(C_{j} \in \mathbb{C}^{r \times n_{j}}, J_{j} \in \mathbb{C}^{n_{j} \times n_{j}}\right)$ is a finite Jordan pair corresponding to the zero $\lambda_{j} \neq 0$ of $A(\sigma)$, then $\tilde{\beta}_{j}(k)=C_{j} J_{j}^{-1}\left(J_{j}^{-1}\right)^{k} x_{0}$ is a solution of (5).

Proof. Since $\left(C_{j}, J_{j}\right)$ is a finite Jordan pair of the polynomial matrix $A(\sigma)$, by Definition 3 it satisfies the equation

$$
A_{q} C_{j} J_{j}^{q}+\cdots+A_{1} C_{j} J_{j}+A_{0} C_{j}=0 .
$$

Now, substituting $\tilde{\beta}_{j}(k)$ into (5), we obtain

$$
\begin{aligned}
& \tilde{A}(\sigma) \tilde{\beta}_{j}(k) \\
& \quad=\tilde{A}(\sigma) C_{j} J_{j}^{-1}\left(J_{j}^{-1}\right)^{k} x_{0} \\
& \quad=A_{0} C_{j}\left(J_{j}^{-1}\right)^{k+q+1} x_{0}+\cdots+A_{q} C_{j}\left(J_{j}^{-1}\right)^{k+1} x_{0} \\
& \quad=\left(A_{q} C_{j} J_{j}^{q}+\cdots+A_{1} C_{j} J_{j}+A_{0} C_{j}\right)\left(J_{j}^{-1}\right)^{k+q+1} x_{0} \\
& \quad \stackrel{700}{=} 0 .
\end{aligned}
$$

Thus, $\tilde{\beta}_{j}(k)$ is a solution of the dual system (5).

In the above theorem, we managed to match a certain forward solution of (2) to a forward solution of (5), a result that we will utilize in the following.

Since the matrix $J_{j}^{-1}$ is not in Jordan form, we can find a non-singular constant matrix $U \in \mathbb{R}^{n_{j} \times n_{j}}$ such that $J_{j}^{-1}=U \tilde{J}_{j} U^{-1}$, where $\tilde{J}_{j}$ is in Jordan form. With this change, the solution of $\tilde{A}(\sigma) \tilde{\beta}(k)=0$ can also be written as follows:

$$
\begin{aligned}
\tilde{\beta}(k) & =C_{j} J_{j}^{-1}\left(J_{j}^{-1}\right)^{k} x_{0} \\
& =C_{j} U \tilde{J}_{j} U^{-1} U\left(\tilde{J}_{j}\right)^{k} U^{-1} x_{0} \\
& =\tilde{C}_{j}\left(\tilde{J}_{j}\right)^{k}\left(U^{-1} x_{0}\right),
\end{aligned}
$$

where $\tilde{C}_{j}=C_{j} U \tilde{J}_{j}$. Therefore, we see that instead of using the matrix pair

$$
\left(C_{j} J_{j}^{-1} \in \mathbb{C}^{r \times n_{j}}, \quad J_{j}^{-1} \in \mathbb{C}^{n_{j} \times n_{j}}\right),
$$


where the matrix $J_{j}^{-1}$ is not in Jordan form, we can use the matrix pair

$$
\begin{aligned}
& \left(\tilde{C}_{j}=C_{j} U \tilde{J}_{j} \in \mathbb{C}^{r \times n_{j}},\right. \\
& \left.\tilde{J}_{j}=U^{-1} J_{j}^{-1} U \in \mathbb{C}^{n_{j} \times n_{j}}\right) .
\end{aligned}
$$

Overall, from Remark 2 as well as Theorems 8 and 10, the connection between the finite and infinite elementary divisors of $A(\sigma)$ as well as the solutions of (2) and its dual system (5) are summarized in Table1.

To sum up our results, in order to construct an AR representation for a certain forward/backward behavior, one must follow Algorithm 1

Algorithm 1. Construction of an AR representation with a given forward and backward behavior (for $\lambda_{j} \neq 0$ ).

Step 1. Transform the finite Jordan pairs $C_{j} \in \mathbb{C}^{r \times n_{j}}$, $J_{j} \in \mathbb{C}^{n_{j} \times n_{j}}$ that correspond to solutions of the form $\beta(k)=C_{j} J_{j}^{k} x_{0}$ (for $\lambda_{j} \neq 0$ ) to the finite Jordan pairs (72) that correspond to solutions of the form $\tilde{\beta}(k)=$ $\tilde{C}_{j}\left(\tilde{J}_{j}\right)^{k}\left(U^{-1} x_{0}\right)$ of the dual system that we are looking for.

Step 2. Construct infinite Jordan pairs of the matrix $A(\sigma)$ as in Theorem 9 These correspond to the finite Jordan pairs of the dual system at $\sigma=0$.

Step 3. Construct the polynomial matrix $\tilde{A}(\sigma)$ using the method presented in Theorem 6

Step 4. Obtain the polynomial matrix $A(\sigma)=\sigma^{q} \tilde{A}(1 / \sigma)$ and thus the AR representation (2) that we are looking for.

It should be mentioned again that $A(\sigma)$ is not the only polynomial matrix satisfying the given behavior. Different choices of $a$ will result in different matrices that are left unimodular equivalent, as mentioned in Remark 1 .

Example 2. We want to construct an AR representation with the following forward and backward solutions:

$$
\beta_{1}(k)=\underbrace{\left(\begin{array}{c}
1 \\
-1
\end{array}\right)}_{\beta_{1,1}} 2^{k}+\underbrace{\left(\begin{array}{c}
2 \\
0
\end{array}\right)}_{\beta_{1,0}} k 2^{k-1},
$$

Table 1. Connection between the solutions of (2) and (5).

\begin{tabular}{|l|l|l|}
\hline$A(\sigma) \beta(k)=0$ & & $\tilde{A}(\sigma) \beta(k)=0$ \\
\hline \hline $\begin{array}{l}\text { forward/backward } \\
\text { solutions }\left(\lambda_{i} \neq 0\right)\end{array}$ & $\Leftrightarrow$ & $\begin{array}{l}\text { forward/backward solu- } \\
\text { tions }\left(\tilde{\lambda}_{i}=\frac{1}{\lambda_{i}} \neq 0\right)\end{array}$ \\
\hline $\begin{array}{l}\text { strictly forward solu- } \\
\text { tions }\left(\lambda_{i}=0\right)\end{array}$ & $\Leftrightarrow$ & $\begin{array}{l}\text { strictly backward solu- } \\
\text { tions }\left(\tilde{\lambda}_{i}=0\right)\end{array}$ \\
\hline $\begin{array}{l}\text { strictly backward solu- } \\
\text { tions }\left(\tilde{\lambda}_{i}=0\right)\end{array}$ & $\Leftrightarrow$ & $\begin{array}{l}\text { strictly forward solutions } \\
\left(\lambda_{i}=0\right)\end{array}$ \\
\hline
\end{tabular}

$$
\beta_{2}(k)=\underbrace{\left(\begin{array}{l}
-1 \\
-1
\end{array}\right)}_{x_{1,1}} \delta(N-k)+\underbrace{\left(\begin{array}{l}
0 \\
3
\end{array}\right)}_{x_{1,0}} \delta(N-k-1) .(74)
$$

First, define the matrix pairs

$$
\begin{aligned}
& C_{1}=\left(\begin{array}{ll}
\beta_{1,0} & \beta_{1,1}
\end{array}\right)=\left(\begin{array}{cc}
2 & 1 \\
0 & -1
\end{array}\right), \quad J_{1}=\left(\begin{array}{ll}
2 & 1 \\
0 & 2
\end{array}\right), \\
& J_{1}{ }^{-1}=\left(\begin{array}{cc}
\frac{1}{2} & -\frac{1}{4} \\
0 & \frac{1}{2}
\end{array}\right)=\underbrace{\left(\begin{array}{cc}
1 & 0 \\
0 & -4
\end{array}\right)\left(\begin{array}{cc}
\frac{1}{2} & 1 \\
0 & \frac{1}{2}
\end{array}\right)\left(\begin{array}{cc}
1 & 0 \\
0 & -\frac{1}{4}
\end{array}\right)}_{U \tilde{J_{1}} U^{-1}} \text {, } \\
& \tilde{C}_{1}=C_{1} U \tilde{J}_{1}=\left(\begin{array}{ll}
1 & 0 \\
0 & 2
\end{array}\right), \\
& C_{2}=\left(\begin{array}{ll}
x_{1,0} & x_{1,1}
\end{array}\right)=\left(\begin{array}{cc}
0 & -1 \\
3 & -1
\end{array}\right), \quad J_{2}=\left(\begin{array}{ll}
0 & 1 \\
0 & 0
\end{array}\right) .
\end{aligned}
$$

The complete matrix pair is

$$
\begin{gathered}
C=\left(\begin{array}{ll}
\tilde{C}_{1} & C_{2}
\end{array}\right)=\left(\begin{array}{cc|cc}
1 & 0 & 0 & -1 \\
0 & 2 & 3 & -1
\end{array}\right), \\
J=\left(\begin{array}{cc}
\tilde{J}_{1} & 0 \\
0 & J_{2}
\end{array}\right)=\left(\begin{array}{cc|cc}
\frac{1}{2} & 1 & 0 & 0 \\
0 & \frac{1}{2} & 0 & 0 \\
\hline 0 & 0 & 0 & 1 \\
0 & 0 & 0 & 0
\end{array}\right) .
\end{gathered}
$$

For $q=1$, the matrix $S_{0}=C$ does not have full column rank. For $q=2$, the matrix

$$
S_{1}=\left(\begin{array}{c}
C \\
C J
\end{array}\right)=\left(\begin{array}{cccc}
1 & 0 & 0 & -1 \\
0 & 2 & 3 & -1 \\
\hline \frac{1}{2} & 1 & 0 & 0 \\
0 & 1 & 0 & 3
\end{array}\right)
$$

has full column rank.

Let $a=1$. Then

$$
\begin{aligned}
\tilde{\mathrm{A}}(\sigma)= & I_{2}-C\left(J-a I_{4}\right)^{-2}\left\{(\sigma-a) V_{2}\right. \\
& \left.+(\sigma-a)^{2} V_{1}\right\},
\end{aligned}
$$

where

$$
\begin{aligned}
\left(V_{1} \mid V_{2}\right) & =\left(\begin{array}{c}
C \\
C\left(J-a I_{4}\right)^{-1}
\end{array}\right)^{-1} \\
& =\left(\begin{array}{cc|cc}
\frac{7}{5} & -\frac{2}{5} & \frac{1}{5} & -\frac{2}{5} \\
-\frac{3}{5} & \frac{1}{10} & -\frac{3}{10} & \frac{1}{10} \\
\frac{8}{15} & \frac{2}{15} & \frac{4}{15} & -\frac{1}{5} \\
\frac{2}{5} & -\frac{2}{5} & \frac{1}{5} & -\frac{2}{5}
\end{array}\right) .
\end{aligned}
$$

The resulting matrix is

$$
\begin{aligned}
& \tilde{A}(\sigma) \\
& =\left(\begin{array}{cc}
\frac{1}{5}\left(6-23 \sigma+22 \sigma^{2}\right) & -\frac{2}{5}\left(-\sigma+\sigma^{2}\right) \\
\frac{3}{5}\left(1-3 \sigma+2 \sigma^{2}\right) & \frac{1}{5}\left(\sigma+4 \sigma^{2}\right)
\end{array}\right) .
\end{aligned}
$$


Therefore, the matrix that we are looking for is

$$
\begin{aligned}
A(\sigma) & =\sigma^{2} \tilde{A}\left(\frac{1}{\sigma}\right) \\
& =\left(\begin{array}{cc}
\frac{1}{5}\left(6 \sigma^{2}-23 \sigma+22\right) & \frac{2}{5}(\sigma-1) \\
\frac{3}{5}\left(\sigma^{2}-3 \sigma+2\right) & \frac{1}{5}(\sigma+4)
\end{array}\right) .
\end{aligned}
$$

Indeed, the vector functions $\beta_{1}(k), \beta_{2}(k)$ are solutions of the system, i.e., they satisfy $A(\sigma) \beta_{i}(k)=0$, for $i=1,2$.

For a different choice of the parameter $a$, for example, $a=-1$, the resulting matrix is

$$
A_{1}(\sigma)=\left(\begin{array}{cc}
\frac{1}{15}\left(6-7 \sigma+2 \sigma^{2}\right) & \frac{2}{45}(1+\sigma) \\
\frac{1}{5}\left(-2-\sigma+\sigma^{2}\right) & \frac{16+\sigma}{15}
\end{array}\right)
$$

and, as expected, the two matrices are left unimodular equivalent, that is,

$$
A(\sigma)=\underbrace{\left(\begin{array}{cc}
\frac{51}{5} & -\frac{4}{5} \\
\frac{18}{5} & \frac{3}{5}
\end{array}\right)}_{U} A_{1}(\sigma),
$$

with $U$ unimodular.

The above algorithm may suffer from computational difficulties in the case where the forward behavior has polynomial vector valued functions (since these are connected with the finite elementary divisors of $A(\sigma)$ at zero). In that case, the Jordan matrix $J$ will have the zero determinant and thus will not be invertible. This problem can easily be surpassed by replacing $\sigma$ with $\sigma+b$ in $A(\sigma)$, where $b$ does not correspond to a zero of the polynomial matrix. This replacement moves all possible zeros of $A(\sigma)$ to non-zero places. The following remark indicates this solution.

Remark 3. (Gohberg et al., 2009, Proof of Theorem 7.3) Let $A(\sigma)$ be a polynomial matrix.

(a) If $(C, J)$ is a finite Jordan pair of $A(\sigma)$ then $(C, J+$ $\left.b I_{n}\right)$ is a finite Jordan pair of $A(\sigma-b)$.

(b) If $\left(C_{\infty}, J_{\infty}\right)$ is an infinite Jordan pair of $A(\sigma)$ then $\left(C_{\infty}, J_{\infty}\left(I_{\mu}+b J_{\infty}\right)^{-1}\right)$ is an infinite Jordan pair of $A(\sigma-b)$.

The use of the above lemma is illustrated with the following example.

Example 3. We want to construct an AR representation of the form (2) with the following forward and backward behavior:

$$
\beta_{1}(k)=\underbrace{\left(\begin{array}{c}
1 \\
-1
\end{array}\right)}_{\beta_{1,1}} \delta(k)+\underbrace{\left(\begin{array}{l}
1 \\
0
\end{array}\right)}_{\beta_{1,0}} \delta(k-1),
$$

$$
\beta_{2}(k)=\underbrace{\left(\begin{array}{c}
-1 \\
-1
\end{array}\right)}_{x_{1,1}} \delta(N-k)+\underbrace{\left(\begin{array}{c}
-1 \\
1
\end{array}\right)}_{x_{1,0}} \delta(N-k-1) .
$$

First, define the matrix pairs

$$
\begin{aligned}
C_{1} & =\left(\begin{array}{ll}
\beta_{1,0} & \beta_{1,1}
\end{array}\right)=\left(\begin{array}{cc}
1 & 1 \\
0 & -1
\end{array}\right), \\
J_{1} & =\left(\begin{array}{ll}
0 & 1 \\
0 & 0
\end{array}\right), \\
C_{2} & =\left(\begin{array}{cc}
-1 & -1 \\
1 & -1
\end{array}\right), \quad J_{2}=\left(\begin{array}{ll}
0 & 1 \\
0 & 0
\end{array}\right) .
\end{aligned}
$$

We observe that the matrix $J_{1}$ is not invertible. Hence, in order to move on, we set $b=2$ and use the pairs

$$
\begin{gathered}
C_{1}^{*}=C_{1}=\left(\begin{array}{cc}
\beta_{1,0} & \beta_{1,1}
\end{array}\right)=\left(\begin{array}{cc}
1 & 1 \\
0 & -1
\end{array}\right), \\
J_{1}^{*}=J_{1}+b I_{2}=\left(\begin{array}{ll}
2 & 1 \\
0 & 2
\end{array}\right), \\
C_{2}^{*}=C_{2}=\left(\begin{array}{cc}
-1 & -1 \\
1 & -1
\end{array}\right), \\
J_{2}^{*}=J_{2}\left(I_{2}+b J_{2}\right)^{-1}=\left(\begin{array}{ll}
0 & 1 \\
0 & 0
\end{array}\right)=J_{2} .
\end{gathered}
$$

Working with the above pairs we construct the matrix $A(\sigma-b)=A(\sigma-2)$. Let also

$$
\begin{gathered}
J_{1}^{*-1}=\left(\begin{array}{cc}
\frac{1}{2} & -\frac{1}{4} \\
0 & \frac{1}{2}
\end{array}\right)=\underbrace{\left(\begin{array}{cc}
1 & 0 \\
0 & -4
\end{array}\right)}_{U} \underbrace{\left(\begin{array}{cc}
\frac{1}{2} & 1 \\
0 & \frac{1}{2}
\end{array}\right)}_{\tilde{J}_{1}} \underbrace{\left(\begin{array}{cc}
1 & 0 \\
0 & -4
\end{array}\right)}_{U^{-1}}, \\
\tilde{C}_{1}=C_{1} U \tilde{J}_{1} \\
=\left(\begin{array}{cc}
1 & 1 \\
0 & -1
\end{array}\right)\left(\begin{array}{cc}
1 & 0 \\
0 & -4
\end{array}\right)\left(\begin{array}{cc}
\frac{1}{2} & 1 \\
0 & \frac{1}{2}
\end{array}\right)=\left(\begin{array}{cc}
\frac{1}{2} & -1 \\
0 & 2
\end{array}\right) .
\end{gathered}
$$

The complete matrix pair is

$$
\begin{gathered}
C=\left(\begin{array}{ll}
\tilde{C}_{1} & C_{2}
\end{array}\right)=\left(\begin{array}{cc|cc}
\frac{1}{2} & -1 & -1 & -1 \\
0 & 2 & 1 & -1
\end{array}\right), \\
J=\left(\begin{array}{cc}
\tilde{J}_{1} & 0 \\
0 & J_{2}
\end{array}\right)=\left(\begin{array}{cc|cc}
\frac{1}{2} & 1 & 0 & 0 \\
0 & \frac{1}{2} & 0 & 0 \\
\hline 0 & 0 & 0 & 1 \\
0 & 0 & 0 & 0
\end{array}\right) .
\end{gathered}
$$

Now start assuming values for $q$. For $q=1$, the matrix $S_{0}=C$ does not have full column rank. For $q=2$, the matrix

$$
S_{2}=\left(\begin{array}{c}
C \\
C J
\end{array}\right)=\left(\begin{array}{cccc}
\frac{1}{2} & -1 & -1 & -1 \\
0 & 2 & 1 & -1 \\
\hline \frac{1}{4} & 0 & 0 & -1 \\
0 & 1 & 0 & 1
\end{array}\right)
$$


has $\operatorname{det}\left(S_{2}\right)=1 / 4 \neq 0$ so it has full rank. Let $a=1$. Then

$$
\begin{aligned}
\tilde{A}^{*}(\sigma)= & I_{2}-C\left(J-a I_{4}\right)^{-2}\left((\sigma-a) V_{2}\right. \\
& \left.+(\sigma-a)^{2} V_{1}\right),
\end{aligned}
$$

where

$$
\left(V_{1} \mid V_{2}\right)=\left(\begin{array}{c}
C \\
C\left(J-a I_{4}\right)^{-1}
\end{array}\right)^{-1}=\left(\begin{array}{cc|cc}
-8 & -2 & -5 & 1 \\
1 & 0 & \frac{1}{2} & -\frac{1}{2} \\
-4 & 0 & -2 & 1 \\
-2 & -1 & -1 & 0
\end{array}\right) .
$$

The resulting matrix is

$$
\tilde{A}^{*}(\sigma)=\left(\begin{array}{cc}
-\sigma+2 \sigma^{2} & -\sigma+\sigma^{2} \\
-1+3 \sigma-2 \sigma^{2} & -1+\sigma+\sigma^{2}
\end{array}\right) .
$$

The matrix $A(\sigma-2)$ is the dual of the above matrix,

$$
\begin{aligned}
A(\sigma-2) & =\sigma^{2} \tilde{A}^{*}\left(\frac{1}{\sigma}\right) \\
& =\left(\begin{array}{cc}
2-\sigma & 1-\sigma \\
-2+3 \sigma-\sigma^{2} & 1+\sigma-\sigma^{2}
\end{array}\right)
\end{aligned}
$$

and the matrix that we were initially searching for is given by substituting $\sigma$ by $\sigma+2$ and is equal to

$$
A(\sigma)=\left(\begin{array}{cc}
-\sigma & -1-\sigma \\
-\sigma-\sigma^{2} & -1-3 \sigma-\sigma^{2}
\end{array}\right)
$$

with

$$
\begin{gathered}
S_{A(\sigma)}^{\mathbb{C}}(\sigma)=\left(\begin{array}{cc}
1 & 0 \\
0 & \sigma^{2}
\end{array}\right), \\
S_{A(\sigma)}^{\infty}(\sigma)=\sigma^{2} S_{\tilde{A}(\sigma)}^{0}\left(\frac{1}{\sigma}\right) \\
=\sigma^{2}\left(\begin{array}{cc}
1 & 0 \\
0 & \frac{1}{\sigma^{2}}
\end{array}\right)=\left(\begin{array}{cc}
\sigma^{2} & 0 \\
0 & 1
\end{array}\right) .
\end{gathered}
$$

As expected, $A(\sigma) \beta_{i}(k)=0$ for $i=1,2$.

\section{Notes on the power of a model}

The notion of power in modeling was introduced by Willems (1986) and later studied by Willems (2007) and Zerz (2008a). The power of a model is defined as the ability of the constructed model to describe the given behavior, i.e., the given data, but as anything little else as possible. Accordingly, this takes place if we define as $B$ the behavior of the system we have constructed, that is, the complete set of vector valued functions which satisfy it:

$$
B=\left\{w:[0, N] \rightarrow \mathbb{R}^{r} \mid A(\sigma) w(k)=0\right\}
$$

or, equivalently,

$$
\mathrm{B}=\operatorname{ker} \mathrm{A}(\sigma),
$$

and we do not simply desire this behavior to include the given functions. This should obviously be the aim of the modeling procedure, but the optimal goal for the constructed model is to have no other behavior, linearly independent from the prescribed. Thus, for any other model with the behavior $B_{1}$ we want

$$
\left\{B \text { more powerful than } B_{1}\right\} \Leftrightarrow\left\{B \subseteq B_{1}\right\} \text {. }
$$

Now, as have mentioned previously, for a given number of vector valued functions, the system created by the proposed algorithms may still include some extra forward/backward behavior if the equation $n+\mu=r q$ is not satisfied. In this case the system model is not the most powerful one (and no such model can be created for a square and regular system matrix).

Theorem 11. Given the following vector valued functions:

$$
\begin{aligned}
\beta_{j}(k)= & \lambda_{j}^{k} \beta_{j, q_{j}-1}+\cdots+\left(\begin{array}{c}
k \\
q_{j}-1
\end{array}\right) \lambda_{j}^{k-q_{j}} \beta_{j, 0}, \\
\beta_{i}(k)= & \delta(k) \beta_{i, p_{i}-1}+\cdots+\delta\left(k-\left(p_{i}-1\right)\right) \beta_{i, 0}, \\
\beta_{p}(k)= & x_{p, \mu_{p}-1} \delta(N-k) \\
& +\cdots+x_{p, 0} \delta\left(N-k-\mu_{p}+1\right),
\end{aligned}
$$

for $j=1, \ldots, m_{0}, i=1, \ldots, m_{1}$ and $p=1, \ldots, m_{2}$, let

$$
n=\sum_{j=1}^{m_{0}} q_{i}+\sum_{i=1}^{m_{1}} p_{i}, \quad \mu=\sum_{p=1}^{m_{2}} \mu_{p} .
$$

The system $A(\sigma) \beta(k)=0$ constructed by the proposed Algorithm 1 corresponding to the behavior $\mathrm{B}=$ $\operatorname{ker} \mathrm{A}(\sigma)$, is the most powerful model that describes the above vector functions iff there exists $q \in \mathbb{N}$ such that 15] holds.

An example where the constructed system is not the most powerful model is given below.

Example 4. We want to construct an AR representation with the following forward and backward solutions:

$$
\begin{aligned}
\beta_{1}(k)= & \underbrace{\left(\begin{array}{c}
1 \\
-1 \\
1
\end{array}\right)}_{\beta_{1,1}}+\underbrace{\left(\begin{array}{l}
2 \\
0 \\
0
\end{array}\right)}_{\beta_{1,0}} k, \\
\beta_{2}(k)= & \underbrace{\left(\begin{array}{c}
-1 \\
-1 \\
0
\end{array}\right)}_{x_{2,2}} \delta(N-k) \\
& +\underbrace{\left(\begin{array}{c}
-1 \\
1 \\
1
\end{array}\right)}_{x_{2,1}} \delta(N-k-1)
\end{aligned}
$$




$$
+\underbrace{\left(\begin{array}{l}
0 \\
3 \\
3
\end{array}\right)}_{x_{2,0}} \delta(N-k-2) .
$$

First, define the matrix pairs

$$
\begin{gathered}
C_{1}=\left(\begin{array}{ll}
\beta_{1,0} & \beta_{1,1}
\end{array}\right)=\left(\begin{array}{cc}
2 & 1 \\
0 & -1 \\
0 & 1
\end{array}\right), \\
J_{1}=\left(\begin{array}{cc}
1 & 1 \\
0 & 1
\end{array}\right), \\
J_{1}^{-1}=\left(\begin{array}{cc}
1 & -1 \\
0 & 1
\end{array}\right)=\underbrace{\left(\begin{array}{cc}
-1 & 0 \\
0 & 1
\end{array}\right)\left(\begin{array}{cc}
1 & 1 \\
0 & 1
\end{array}\right)\left(\begin{array}{cc}
-1 & 0 \\
0 & 1
\end{array}\right)}, \\
\tilde{C}_{1}=C_{1} U \tilde{J}_{1}=\left(\begin{array}{cc}
-2 & -1 \\
0 & -1 \\
0 & 1
\end{array}\right), \\
J_{2}=\left(\begin{array}{ccc}
0 & -1 & -1 \\
3 & 1 & 0 \\
0 & 0 & 1 \\
0 & 0 & 0
\end{array}\right) .
\end{gathered}
$$

The complete matrix pair is $C=\left(\begin{array}{ll}\tilde{C}_{1} & C_{2}\end{array}\right), J=$ $\operatorname{blockdiag}\left(\tilde{J}_{1}, J_{2}\right)$.

Before we start assuming values for $q$, we see that for the functions given above, we have $n=2, \mu=3$ and since we want to create a square regular system, $r=3$. However, we easily observe that there is no $q$ that satisfies

$$
n+\mu=r q \Rightarrow 5=3 q \text {. }
$$

Hence, we expect the final system to include undesired solutions.

For $q=1$, the matrix $S_{0}=C$ does not have full column rank. For $q=2$, the matrix $S_{1}=$

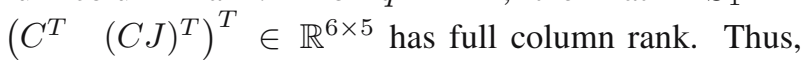
let $a=2$ and

$$
\begin{aligned}
\tilde{\mathrm{A}}(\sigma)= & I_{3}-C\left(J-a I_{5}\right)^{-2}\left\{(\sigma-a) V_{2}\right. \\
& \left.+(\sigma-a)^{2} V_{1}\right\},
\end{aligned}
$$

where $\left(V_{1} V_{2}\right)$ is the generalized inverse of

$$
V=\left(\begin{array}{ll}
V_{1} & V_{2}
\end{array}\right)=\left(\begin{array}{c}
C \\
C\left(J-a I_{5}\right)^{-1}
\end{array}\right)^{-1}
$$

Computing $\tilde{A}(\sigma)$ and after some simplifications we obtain the matrix we are looking for:

$$
A(\sigma)=\sigma^{2} \tilde{A}\left(\frac{1}{\sigma}\right)
$$

$$
\begin{aligned}
= & \left(\begin{array}{cc}
1084-1996 \sigma+912 \sigma^{2} & 562-1590 \sigma+932 \sigma^{2} \\
714-2142 \sigma+1428 \sigma^{2} & 347-775 \sigma+1418 \sigma^{2} \\
-102+306 \sigma-204 \sigma^{2} & 399-1235 \sigma+874 \sigma^{2} \\
& -714+1894 \sigma-932 \sigma^{2} \\
& -271+1251 \sigma-1418 \sigma^{2} \\
& -51+1167 \sigma-874 \sigma^{2}
\end{array}\right)
\end{aligned}
$$

with Smith forms

$$
\begin{aligned}
& S_{A(\sigma)}^{\mathbb{C}}(\sigma)=\left(\begin{array}{ccc}
1 & 0 & 0 \\
0 & 1 & 0 \\
0 & 0 & (\sigma-1)^{2}(277 \sigma-60)
\end{array}\right), \\
& S_{\tilde{A}(\sigma)}^{0}(\sigma)=\left(\begin{array}{ccc}
1 & 0 & 0 \\
0 & 1 & 0 \\
0 & 0 & \sigma^{3}
\end{array}\right) .
\end{aligned}
$$

As expected, the final system matrix $A(\sigma)$ has an extra finite elementary divisor at $\sigma=60 / 277$. This gives rise to a third solution vector for the system, linearly independent of $\beta_{1}(k)$ and $\beta_{2}(k)$. Using the method proposed by Karampetakis (2004), we find that the third solution is

$$
\beta_{3}(k)=\left(\begin{array}{c}
-\frac{21296}{59149} \\
\frac{477249619}{1409047478} \\
-\frac{654963505}{1409047478}
\end{array}\right)\left(\frac{60}{277}\right)^{k}
$$

Hence, the above system $A(\sigma) \beta(k)=0$ is not the most powerful model describing the vectors $\beta_{1}(k)$ and $\beta_{2}(k)$. A construction of a non-regular system that satisfies $\beta_{1}(k), \beta_{2}(k)$ is still possible, for example, the system with matrix

$$
A(\sigma)=\left(\frac{7}{2}-\frac{9}{2} \sigma+\sigma^{4} \quad 1-\sigma^{5} \quad \sigma^{5}\right)
$$

is non-regular and satisfies $A(\sigma) \beta_{i}(k)=0$. What must be noted, though, is that solutions of non-regular systems can either be connected to their f.e.d.'s and i.e.d.'s or the structure of the right null space, since non-regular systems have an infinite number of forward and backward propagating solutions, due to the right null space of $A(\sigma)$, as evidenced by Karampetakis (2004). Therefore, in this case, too, the constructed system includes an additional undesired behavior.

\section{Conclusions}

We have proposed an algorithm for constructing a regular AR representation which satisfies given forward and backward behaviors. This is a discrete time analog of the work by Karampetakis (2015), where the problem was formulated for continuous time AR representations as well as the modeling of the smooth and impulsive system behavior. Our further aim is to extend this theory to the 
case of non-regular AR representations, since we have noticed that for a given forward-backward behavior we can always achieve a non-regular AR representation that satisfies this behavior.

\section{Acknowledgment}

The authors wish to thank the anonymous reviewers for their insightful comments that significantly improved the quality of this paper.

\section{References}

Antoniou, E., Vardulakis, A. and Karampetakis, N. (1998). A spectral characterization of the behavior of discrete time AR-representations over a finite time interval, Kybernetika 34(5): 555-564.

Antoulas, A. and Willems, J. (1993). A behavioral approach to linear exact modeling., IEEE Transactions on Automatic Control 38(12): 1776-1802.

Antsaklis, P.J. and Michel, A.N. (2006). Linear Systems, 2nd Edn., Birkhäuser, Boston, MA.

Bernstein, D.S. (2009). Matrix Mathematics. Theory, Facts, and Formulas, 2nd Edn., Princeton University Press, Princeton, NJ.

Campbell, S. (1980). Singular Systems of Differential Equations, Vol. 1, Research Notes in Mathematics, Pitman, London.

Gantmacher, F.R. (1959). The Theory of Matrices, Vols. 1, 2, Chelsea Publishing Co., New York, NY.

Gohberg, I., Lancaster, P. and Rodman, L. (2009). Matrix Polynomials, Reprint, SIAM, Philadelphia, PA.

Hayton, G., Pugh, A. and Fretwell, P. (1988). Infinite elementary divisors of a matrix polynomial and implications, International Journal of Control 47(1): 53-64.

Kaczorek, T. (2007). Polynomial and Rational Matrices. Applications in Dynamical Systems Theory, Springer, Dordrecht.

Kaczorek, T. (2014). Minimum energy control of fractional descriptor positive discrete-time linear systems, International Journal of Applied Mathematics and Computer Science 24(4): 735-743, DOI: 10.2478/amcs-2014-0054.

Kaczorek, T. (2015). Analysis of the descriptor Roesser model with the use of the Drazin inverse, International Journal of Applied Mathematics and Computer Science 25(3): 539-546, DOI: 10.1515/amcs-2015-0040.

Karampetakis, N.P. (2004). On the solution space of discrete time AR-representations over a finite time horizon, Linear Algebra and Its Applications 382: 83-116.

Karampetakis, N.P. (2015). Construction of algebraic-differential equations with given smooth and impulsive behaviour, IMA Journal of Mathematical Control and Information 32(1): 195-224.

Karampetakis, N.P. and Vologiannidis, S. (2003). Infinite elementary divisor structure-preserving transformations for polynomial matrices, International Journal of Applied Mathematics and Computer Science 13(4): 493-503.

Karampetakis, N., Vologiannidis, S. and Vardulakis, A. (2004). A new notion of equivalence for discrete time AR representations, International Journal of Control 77(6): 584-597.

Markovsky, I., Willems, J.C., Van Huffel, S. and De Moor, B. (2006). Exact and Approximate Modeling of Linear Systems. A Behavioral Approach, SIAM, Philadelphia, PA.

Praagman, C. (1991). Invariants of polynomial matrices, Proceedings of the 1st European Control Conference, Grenoble, France, pp. 1274-1277.

Vardulakis, A. (1991). Linear Multivariable Control. Algebraic Analysis and Synthesis Methods, John Wiley \& Sons, Chichester.

Vardulakis, A., Limebeer, D. and Karcanias, N. (1982). Structure and Smith-MacMillan form of a rational matrix at infinity, International Journal of Control 35(4): 701-725.

Willems, J.C. (1986). From time series to linear system, II: Exact modelling, Automatica 22(6): 675-694.

Willems, J.C. (1991). Paradigms and puzzles in the theory of dynamical systems, IEEE Transansactions on Automatic Control 36(3): 259-294.

Willems, J.C. (2007). Recursive computation of the MPUM, in A. Chiuso et al. (Eds.), Modeling, Estimation and Control, Springer, Berlin, pp. 329-344.

Zaballa, I. and Tisseur, F. (2012). Finite and infinite elementary divisors of matrix polynomials: A global approach, MIMS EPrint 2012.78, Manchester Institute for Mathematical Sciences, University of Manchester, Manchester.

Zerz, E. (2008a). Behavioral systems theory: A survey, International Journal of Applied Mathematics and Computer Science 18(3): 265-270, DOI: 10.2478/v10006-008-0024-9.

Zerz, E. (2008b). The discrete multidimensional MPUM, Multidimensional System Signal Processing 19(3-4): 307-321.

Zerz, E., Levandovskyy, V. and Schindelar, K. (2011). Exact linear modeling with polynomial coefficients, Multidimensional System Signal Processing 22(1-3): 55-65.

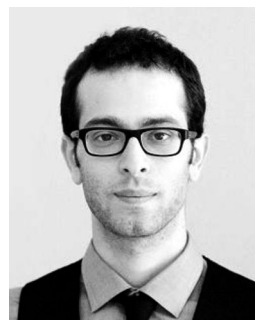

and $\mathrm{LT}_{\mathrm{E}} \mathrm{X}$.
Lazaros Moysis is a PhD student at the Department of Mathematics, Aristotle University of Thessaloniki, Greece. He received a Bachelor's degree in 2011 and a Master's degree in 2013. His research interests include the theory of control systems, linear systems, descriptor and higher order systems of differential or difference equations, as well as matrix theory. He has published works in international journals and conferences, as well as some free tutorials on Matlab 


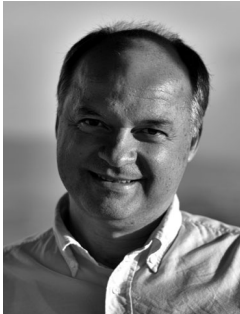

Nicholas P. Karampetakis was born in Drama, Greece, in 1967. He received the Bachelor's and $\mathrm{PhD}$ degrees in mathematics from the School of Mathematics, Aristotle University of Thessaloniki, Greece, in 1989 and 1993, respectively. Since August 2014 he has been a professor of mathematical theory of control systems with the School of Mathematics, Aristotle University of Thessaloniki, and since September 2013 he has been the head of that school. His research interests lie mainly in algebraic methods for computer aided design of control systems (CACSD), numerical and symbolic algorithms for CACSD, polynomial matrix theory and issues related to mathematical systems theory. He is a senior member of the IEEE and a vice-chair of the IEEE Action Group on Symbolic Methods for CACSD. He is a member of the Technical Committee on Linear Control Systems of the International Federation of Automatic Control (IFAC). He is also an associate editor of the Journal of Multidimensional Systems and Signal Processing, Systems Science and Control Engineering, and the International Journal of Circuits, Systems and Signal Processing.

Received: 25 May 2016

Revised: 14 September 2016

Accepted: 12 October 2016 